\title{
Efecto de la radiación ultravioleta-B sobre el crecimiento y la fotosíntesis de siete variedades de trigo (Triticum aestivum L.)
}

\author{
Effect of the ultraviolet-B radiation on growth and photosynthesis \\ of seven varieties of wheat (Triticum aestivum $L$.)
}

MANUEL PINTO', CAROLINA LIZANA, CLAUDIO PASTENES, ALEJANDRO RIQUELME \& MARITZA BERTI

Universidad de Chile, Facultad de Ciencias Agropecuarias, Casilla 1004, Santiago, Chile, e-mail : 'manpinto@ abello.dic.uchile.cl

\begin{abstract}
RESUMEN
Con el fin de determinar la sensibilidad a la radiación UV-B de variedades de trigo cultivadas en Chile, plantas de las variedades ERP- V8, Lilén, Millaleu, Nobo, Reihue, Talhuén y Huayún, fueron irradiadas durante las seis primeras semanas de crecimiento con $22,5 \mu \mathrm{W} \mathrm{cm}-2$ de radiación $U V-B$, durante 4 horas diarias y bajo una intensidad de radiación visible promedio de $400 \mu \mathrm{mol} \mathrm{m} \mathrm{s}^{-1}$ ( $12 \mathrm{~h}$ de fotoperíodo). En función de la respuesta en crecimiento (peso fresco y seco y área foliar) al tratamiento UV-B, las variedades Talhuén Lilén y Nobo, resultaron ser los más sensibles. En todas las variedades la cantidad de compuestos absorbedores de UV-B aumentó como resultado del tratamiento con radiación UV-B. Sin embargo, no se observó una relación clara entre este aumento y la sensibilidad de las variedades a esta radiación. La asimilación neta de $\mathrm{CO}_{2}$ se vio negativamente afectada en todas las variedades, pero este efecto fue significativamente mayor en aquellas clasificadas como más sensibles. El rendimiento cuántico del PSII medido como Fv/Fm no se afectó por la acción del tratamiento UV-B, pero claramente disminuyó en todas las variedades luego de someter éstas a una alta intensidad de radiación visible. Este efecto fotoinhibitorio de la luz visible fue menos marcado en las plantas previamente irradiadas con UV-B, especialmente en las variedades más sensibles (Talhuén y Lilén). El efecto fotoprotector ejercido por el tratamiento previo con radiación UV-B se discute en el presente trabajo. Finalmente se concluye que las variedades ERP-V8, Millaleu y Huayún resultaron ser las más tolerantes al nivel de radiación UVB impuesto.
\end{abstract}

Palabras clave: radiación UV - B, fotosíntesis, crecimiento, variedades de trigo, Triticum aestivum $\mathrm{L}$.

\begin{abstract}
The effect of UV-B radiation on growth and photosynthesis of seven Chilean varieties of wheat ( ERP V-8, Lilén, Millaleu, Nobo, Reihue, Talhuén and Huayún) was evaluated during the first six weeks of growth. Plants were irradiated 4 hours daily with $22.5 \mu \mathrm{W} \mathrm{cm} \mathrm{cm}^{-2}$ of UV-B radiation and with a white light intensity (12 h photoperiod) that averaged $400 \mu \mathrm{mol} \mathrm{m} \mathrm{m}^{-2} \mathrm{~s}^{-1}$. The varieties Talhuén, Lilén and Nobo were the most sensitive to this treatment in terms of their reduction in leaf area and in fresh and dry matter accumulation. UV-B absorbing compounds increased in all the plants treated with UV-B but this increase was not related with the growth sensitivity shown by the different varieties to this radiation. A decrease in $\mathrm{CO}_{2}$ assimilation was detected in all the varieties as a consequence of the UV-B treatment. However, this decrease was significant only in Talhuén which was one of the most sensitive to UV-B. The PSII quantum yield, measured as Fv/Fm ratio, decreased in all the varieties under high white light intensities. This photoinhibitory effect was reduced when plants were previously irradiated with UV-B, particularly in the more sensitive varieties (Talhuén y Lilén). This kind of photoprotection exerted by the UV-B radiation is discussed. Finally, it is concluded that varieties ERP-V8, Millaleu and Huayún were the most tolerant to the level of UV-B radiation used in this case.
\end{abstract}

Key words: UV-B radiation, photosynthesis, growth, wheat varieties, Triticum aestivum $\mathrm{L}$. 


\section{INTRODUCCION}

La disminución de la concentración de ozono estratosférico, causada por la contaminación industrial, ha provocado durante las últimas décadas un significativo aumento de la radiación ultravioleta-B (UV-B) a nivel de la tropósfera (Frederick et al. 1989). Este aumento de radiación UV-B ha sido particularmente importante en la zona austral, cercana a la Antártica, en donde las pérdidas de ozono registradas entre 1969 y 1986 superarían el 50\% (Kirchhoff et al. 1997). Estimaciones hechas por Roy et al. (1990), indican que una disminución del $1 \%$ en la concentración total de ozono provocaría un aumento promedio del $2 \%$ en la radiación UV-B incidente en la superficie terrestre. Este valor aunque pequeño respecto de la proporción de otras longitudes de ondas, podría no tener efecto en organismos de latitudes bajas o de grandes altitudes normalmente adaptados a altas intensidades de UV-B. Sin embargo, en organismos adaptados a intensidades moderadas o bajas, es posible que el efecto sea importante principalmente por la acción de esta radiación sobre los ácidos nucleicos, los pigmentos y otras biomoléculas (Nedunchezhian \& Kulandaivelu 1991, Strid et al. 1994, Fiscus \& Booker 1995, Jansen et al. 1998). En los vegetales, la adaptación a diferentes intensidades de esta radiación ha sido de especial importancia para su evolución y desarrollo en distintos hábitat. Variaciones en el grado de tolerancia entre especies han sido bien documentadas (Bornman \& Voguelman 1991, Day et al. 1992, Teramura 1990) y una clasificación general permite señalar que las especies anuales son más sensibles a la radiación UV que las perennes (Day 1993). Igualmente, las leguminosas lo serían más que las gramineas (Teramura 1983). Entre genotipos o variedades de especies cultivadas, también se han encontrado importantes variaciones en la sensibilidad a esta radiación (Teramura \& Sullivan 1987, Teramura et al. 1991, Huang et al. 1993, Miller et al. 1994). Esto tiene especial importancia para el mejoramiento genético de los principales cultivos respecto de este factor, como también para obtener información sobre los mecanismos responsables del grado de sensibilidad o tolerancia a la radiación UV-B en los vegetales. El grado de sensibilidad a esta radiación, en gran parte de los casos, se ha detectado por su efecto en procesos que, como la fotosíntesis, son claves para el crecimiento y desarrollo de los vegetales. Numerosos son los estudios que indican que la capacidad fotosintética puede ser alterada debido a la acción directa de la radiación UV-B sobre las enzimas fotosintéticas más importantes (Sullivan \&
Teramura 1990, Strid et al. 1990, Ziska \& Teramura 1992, Fiscus \& Booker 1995), como también por su acción sobre el Fotosistema II (PSII) (Bornman \& Voguelman 1991, Teramura et al. 1991, Huang et al. 1993, Ziska et al. 1993), en particular sobre la degradación de la proteína D1 (Bornman 1989, Nedunchezhian \& Kulandaivelu 1991, Strid et al. 1994). Indirectamente la radiación UV-B también puede alterar la fotosíntesis por sus efectos sobre la función estomática (Day \& Voguelman 1995, Zenthen et al. 1997), sobre el área foliar (Tevini et al. 1981, Kulandaivelu et al. 1989, Pinto et al. 1999), como también sobre el contenido de diversos pigmentos, principalmente flavonoides y clorofilas (Tevini et al. 1991, Strid \& Porra 1992, Huang et al. 1993, Wilson \& Greenberg 1993, Teramura \& Sullivan 1994, Day et al. 1996). Frente a incrementos en la radiación UV-B, se ha visto que variedades de poroto más sensibles, experimentan aumentos poco significativos en la concentración de compuestos absorbedores de UV-B y reducciones importantes en el área foliar y la asimilación neta de $\mathrm{CO}_{2}$. Estos cambios finalmente redundan en una disminución en la acumulación de biomasa (Pinto et al. 1999) lo cual también ha sido observado en otras especies cultivadas (Teramura, 1990, Feldhein \& Conner 1996). En trigo por ejemplo, existen varios estudios que señalan efectos de la radiación UV-B similares a los indicados anteriormente (Becwar et al. 1982, Kulandaivelu et al. 1989, Sharma et al. 1998). Esta especie, por poseer una amplia distribución a través de los más diversos hábitat en el mundo, se piensa debiera tener numerosos genotipos o variedades con diversos grados de sensibilidad a esta radiación (Teramura et al. 1991). En este trabajo se postula que esta variabilidad debiera también reflejarse en las variedades comerciales cultivadas en Chile y cuyo cultivo se distribuye desde el Norte a la zona Centro - Sur del país a través de un importante gradiente de intensidad de radiación UV-B (Cabrera et al. 1995). Esta distribución de variedades comerciales, tradicionalmente se ha establecido en función de diferentes factores ambientales en que la radiación solar, en particular la radiación UV-B, no ha sido directamente considerada. Debido a esto es muy posible que variedades recomendadas para una amplia distribución, posean grados de sensibilidad distintos a esta radiación. Dado que para las variedades de trigo chilenas no existe información a este respecto y que antecedentes en tal sentido podrían ser de utilidad tanto para la determinación de una mejor distribución varietal como para el mejoramiento de la especie respecto de este carácter, el principal objetivo de este trabajo fue comparar la respuesta al incre- 
mento de la radiación UV-B en siete variedades recomendadas para la zona comprendida entre la III $\left(28^{\circ} \mathrm{S}\right)$ y la VIII Región $\left(38^{\circ} \mathrm{S}\right)$ a través de la medición de parámetros de crecimiento y fotosintéticos durante los primeros estados de desarrollo.

\section{MATERIALES Y METODOS}

\section{Material vegetal y condiciones del ensayo}

Semillas de trigo prehidratadas (a $25^{\circ} \mathrm{C} / 24 \mathrm{~h}$ ) de las variedades: ERP V-8, Lilén, Millaleu, Nobo, Reihue, Talhuén y Huayún, se sembraron en bolsas de plástico negro $(650 \mathrm{cc})$ conteniendo una mezcla de vermiculita y arena $(2: 1 \mathrm{v} / \mathrm{v})$. Todas estas variedades poseen una amplia distribución y son recomendadas por el Instituto de Investigación Agropecuaria (INIA) para ser cultivadas desde la III $\left(26^{\circ} \mathrm{S}\right)$ hasta la VIII región $\left(38^{\circ} \mathrm{S}\right)$. Las plantas ( 7 por bolsa) se cultivaron en un invernadero de vidrio con control de temperatura (20-30\% $/ 10-15^{\circ} \mathrm{C}$ día/noche) hasta el estado de quinta hoja (aproximadamente 6 semanas). En este estado se efectuaron la mayoría de las mediciones. El riego se efectuó con una frecuencia promedio de dos días con $50 \mathrm{ml}$ de solución Hoagland II (Hoagland \& Arnon 1950). La radiación fotosintéticamente activa (PAR) se obtuvo con lámparas de Haluro Metálico (Phillips 400W) y se mantuvo relativamente constante en 400

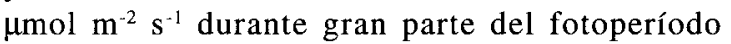
(12 horas) con un máximo al medio día solar cercano a $600 \mu \mathrm{mol} \mathrm{m} \mathrm{m}^{-2} \mathrm{~s}^{-1}$. Desde la emergencia hasta el estado de quinta hoja, seis semanas aproximadamente, un grupo de plantas fueron irradiadas diariamente con radiación UV-B, por un período de 4 horas centrado al mediodía solar. La intensidad de la radiación UV-B (280-320 nm), medida con un radiómetro UV (Solar Light Co, USA) y suministrada por lámparas (Q-Panel 313, Cleveland, USA) fue equivalente a 22,5 $\mu \mathrm{W} \mathrm{cm} \mathrm{cm}^{-2}(280-320 \mathrm{~nm})$. Las lámparas UV se envolvieron en acetato de celulosa (Mica $0,08 \mathrm{~mm}$ ) con el fin de eliminar la radiación UV-C y se ubicaron entre las plantas y lámparas de luz actínica en un dispositivo móvil que permitió mantener la misma intensidad de radiación a medida que las plantas crecían. Otro grupo de plantas no recibió radiación UV-B y en este caso las lámparas UV se envolvieron con poliester de 0,18 mm (Mylar, Dupont, USA) con el fin de eliminar la radiación con longitudes de onda menores a $320 \mathrm{~nm}$. Ambos tipos de filtros se cambiaron cada semana.

\section{Parámetros de crecimiento}

Las plantas se cosecharon al estado de $5^{\mathrm{a}}$ hoja y se les determinó el área foliar y el peso fresco y seco de la parte aérea (hojas + tallos) y de la raíz. El peso seco, se obtuvo secando el material vegetal a $70^{\circ} \mathrm{C}$ por 48 horas, en una estufa con circulación de aire (Memmert U30). El área foliar se calculó cada siete días, midiendo el largo y el ancho de cada hoja por medio de la ecuación obtenida de la regresión lineal entre el producto del largo por el ancho de las hojas y la respectiva área medida con un determinador de área (LI-COR 3000). La ecuación que definió esta relación fue:

$$
\mathrm{AF}=0,428+0,762 \cdot \mathrm{PF} \quad\left(\mathrm{r}^{2}=0,98\right)
$$

donde $\mathrm{AF}$ es el área foliar $\left(\mathrm{cm}^{2}\right)$ y $\mathrm{PF}$ el producto del largo de la hoja por su ancho. El número de determinaciones usadas para establecer esta correlación fue 70 .

Asimilación de $\mathrm{CO}_{2}$ y Fluorescencia de la clorofila " $a$ "

La asimilación de $\mathrm{CO}_{2}$ se midió en la quinta hoja de cada planta inserta en una cámara tipo Parkinson (Parkinson et al. 1980), con control de temperatura por circulación de agua y conectada a un analizador infrarrojo de gases (ADC, UK). La concentración del $\mathrm{CO}_{2}$ en el circuito de referencia fue de $350 \mathrm{ppm}$ y la temperatura al interior de la cámara $25^{\circ} \mathrm{C}$. Las mediciones se realizaron a la misma intensidad luminosa en la cual las plantas fueron cultivadas $\left(400 \mu \mathrm{mol}\right.$ quanta $\left.\mathrm{m}^{-2} \mathrm{~s}^{-1}\right) \mathrm{y}$ los valores se registraron una vez alcanzada la estabilidad de la señal. La emisión de fluorescencia de la clorofila "a" se determinó en la $5^{\mathbf{a}}$ hoja, utilizando un fluorómetro no modulado portátil (PEA, Hansatech, U.K.). El rendimiento fotoquímico del PSII se estimó de acuerdo con Genty et al. (1989) por medio de la obtención del parámetro Fv/Fm en hojas previamente mantenidas en oscuridad por espacio de 30 minutos (Krause \& Weis 1984). Luego de estas mediciones, efectuadas en las plantas cultivadas bajo 400 $\mu \mathrm{mol} \mathrm{m}^{-2} \mathrm{~s}^{-1}$, las plantas se sometieron por 30 minutos a una alta intensidad de radiación natural (aproximadamente $1700 \mu \mathrm{mol} \mathrm{m} \mathrm{m}^{-2} \mathrm{~s}^{-1}$ ), al término del cual se midió nuevamente la relación $\mathrm{Fv} / \mathrm{Fm}$.

\section{Compuestos absorbedores de UV-B}

Estos compuestos se determinaron espectrofotométricamente en extractos brutos de hoja, de 
acuerdo al método descrito por Wilson \& Greenberg (1993). De cada planta se tomó una muestra de hoja de $3 \mathrm{~cm}^{2}$ (9 discos de $0,33 \mathrm{~cm}^{2}$ cada uno) la cual se incubó durante 24 horas $\left(4^{\circ} \mathrm{C}\right)$ con $2,5 \mathrm{ml}$ de una solución de metanol acidificada (metanol $70 \%$ [v/v], $120 \mathrm{mM} \mathrm{HCl}$ ). Luego de la extracción, las muestras se centrifugaron a 5000 $\mathrm{g}$ por $10 \mathrm{~min}$ a temperatura ambiente. La absorbancia medida en los sobrenadantes a 300 nm se utilizó para la estimación de la cantidad de compuestos absorbedores de UV-B. Estos compuestos incluyen principalmente flavonoides $y$ compuestos fenólicos con un máximo de absorción en esta longitud de onda.

\section{Análisis estadístico y diseño experimental}

Para el análisis estadístico, se empleó un diseño de bloques completamente aleatorizados con estructura factorial $2 \times 7$ con 4 repeticiones. Un factor fue el nivel de radiación UV-B aplicado y el otro, los siete genotipos de trigo utilizados. Dado el reducido espacio para efectuar las irradiaciones con UV-B los bloques o replicaciones correspondieron a distintas fechas de siembra. Los resultados se analizaron mediante análisis de varianza y prueba de comparación múltiple de Duncan cuando fue necesario identificar diferencias entre tratamientos específicos.

\section{RESULTADOS}

\section{Efectos sobre el crecimiento}

La evolución del área foliar durante las primeras cuatro semanas del estudio fue bastante similar en todas las variedades y hasta el término de este período, no hubo diferencias entre las plantas irradiadas y las no irradiadas con UV-B (resultados no mostrados). Sin embargo, a partir de la cuarta semana en la variedad Lilén y de la quinta en las variedades Nobo y Talhuén, la tasa de expansión foliar empezó a disminuir en las plantas tratadas con UV-B lo que se tradujo, al final de la $6^{\mathrm{a}}$ semana, en una reducción significativa del área foliar de estos tres genotipos (Fig. 1). Los valores relativos de disminución del área foliar en las variedades Lilén, Talhuén y Nobo fueron $17,5 \%, 18,8 \%$ y $20 \%$ respectivamente. Estos valores resultaron ser bastante más elevados que la reducción promedio $(11,8 \%)$, calculada al considerar el resto de las variedades usadas en el experimento y que no presentaron diferencias al término del período.
La reducción en área foliar fue acompañada por una reducción similar en el peso fresco en las mismas variedades ya mencionadas (Fig. 2). En el caso de la variedad Lilén, esta disminución fue significativa sólo en la parte aérea y total, en cambio en el caso de Nobo y Talhuén los efectos de la irradiación con UV-B fueron también importantes en las raíces. Nobo y Talhuén fueron las variedades más afectadas y la reducción en peso fresco total alcanzó $20,6 \%$ y $20,8 \%$ respectivamente. En Lilén esta reducción fue de $17,7 \%$. Estos valores contrastan con las reducciones observadas en el resto de las variedades en donde en promedio sólo fue $6,4 \%$.

En este sentido las variedades ERP V-8 y Millaleu aparecen con la menor variación en este parámetro y también son ellas, junto con Huayún, las que presentan la menor variación en el peso seco (Fig. 3). Por otra parte, aunque en general en el resto de las variedades la irradiación con UV$B$ induce una disminución del peso seco, esta disminución sólo alcanza significancia estadística en la variedad Nobo. En esta variedad la reducción en peso seco de las plantas irradiadas con

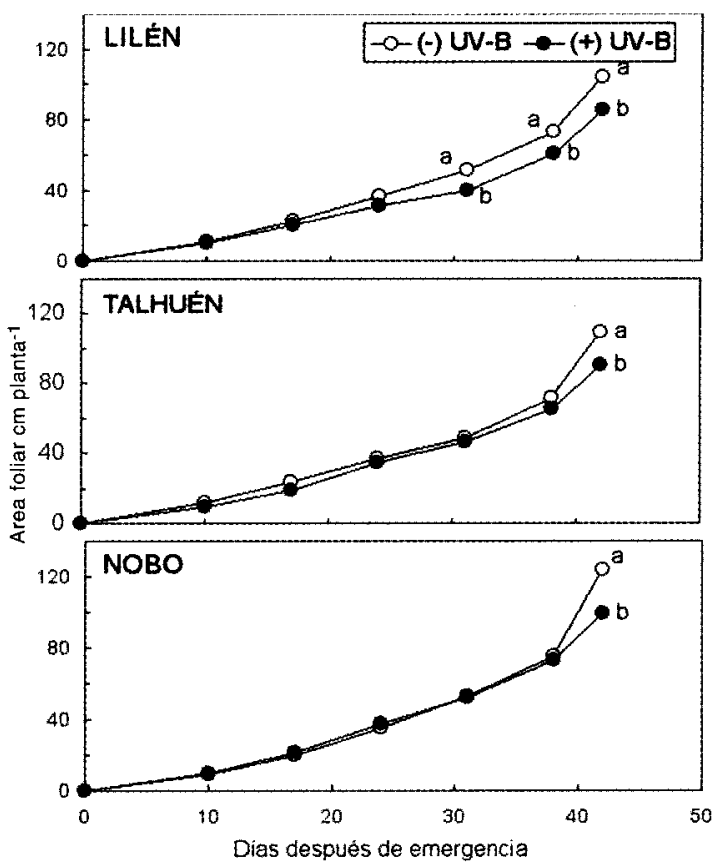

Fig. 1. Efecto de la radiación UV-B sobre el desarrollo del área foliar de las variedades de trigo

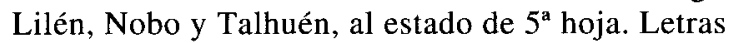
distintas indican diferencias significativas entre tratamientos $(\mathrm{P}<0,05 ; \mathrm{n}=28)$.

Effect of the UV-B irradiation on leaf area of the wheat varieties Lilén, Nobo and Talhuén, at the $5^{\text {th }}$ leaf stage. Different letters, indicate significant differences between treatments $(\mathrm{P}<0.05 ; \mathrm{n}=28)$. 


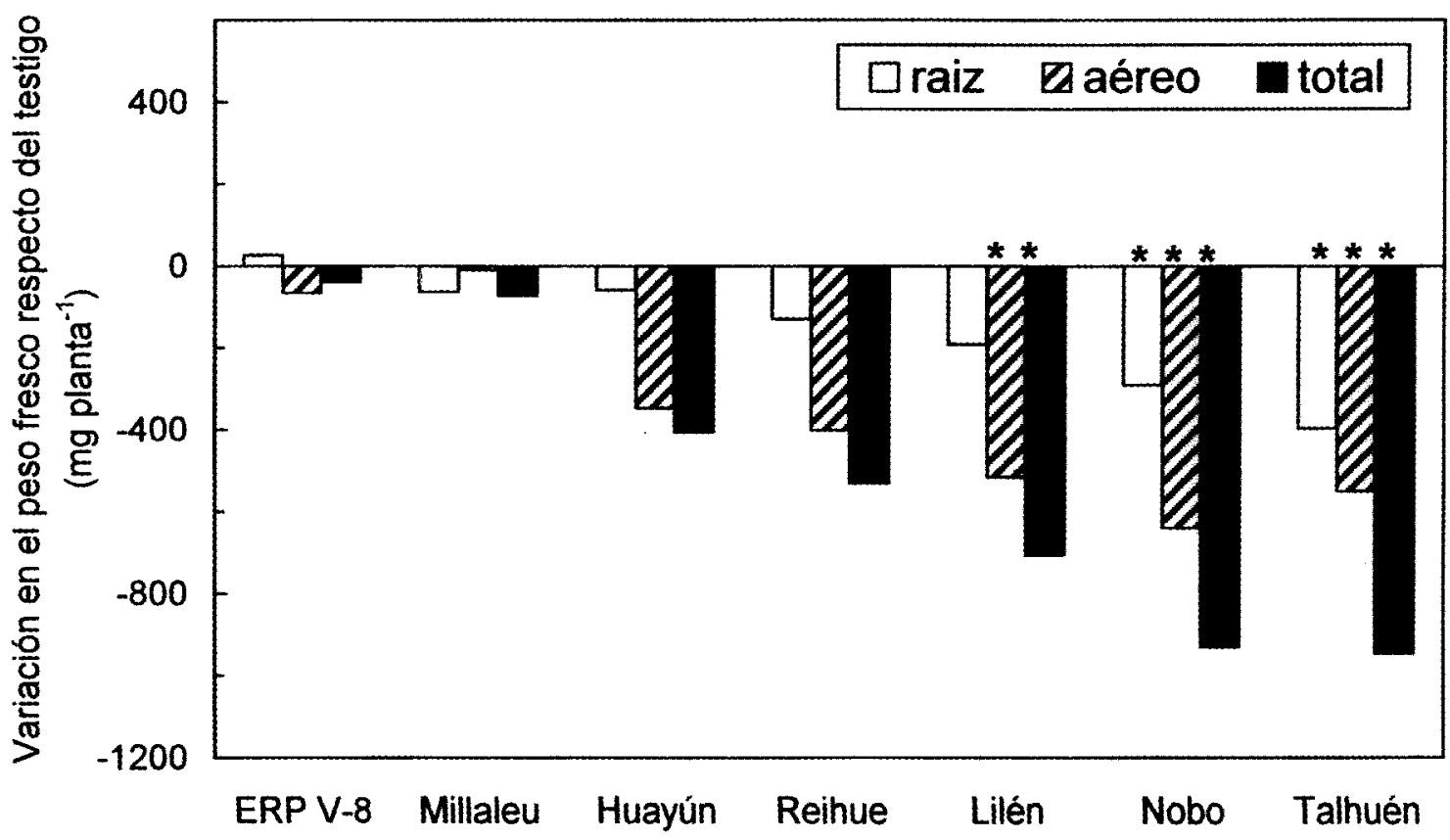

Fig. 2. Efecto de la radiación UV-B sobre la variación del peso fresco de siete variedades de trigo al estado de $5^{a}$ hoja. Los asteriscos indican que la reducción en el peso de las plantas tratadas respecto de los testigos fue significativa $(\mathrm{P}<0,05 ; \mathrm{n}=28)$.

Effect of the UV-B irradiation on the variation of the fresh weight of seven wheat varieties, at the $5^{\text {th }}$ leaf stage. Asterisks indicate significant reductions in weight of UV-B treated plants with respect to non UV-B treated plants $(P<0.05 ; n=8)$.

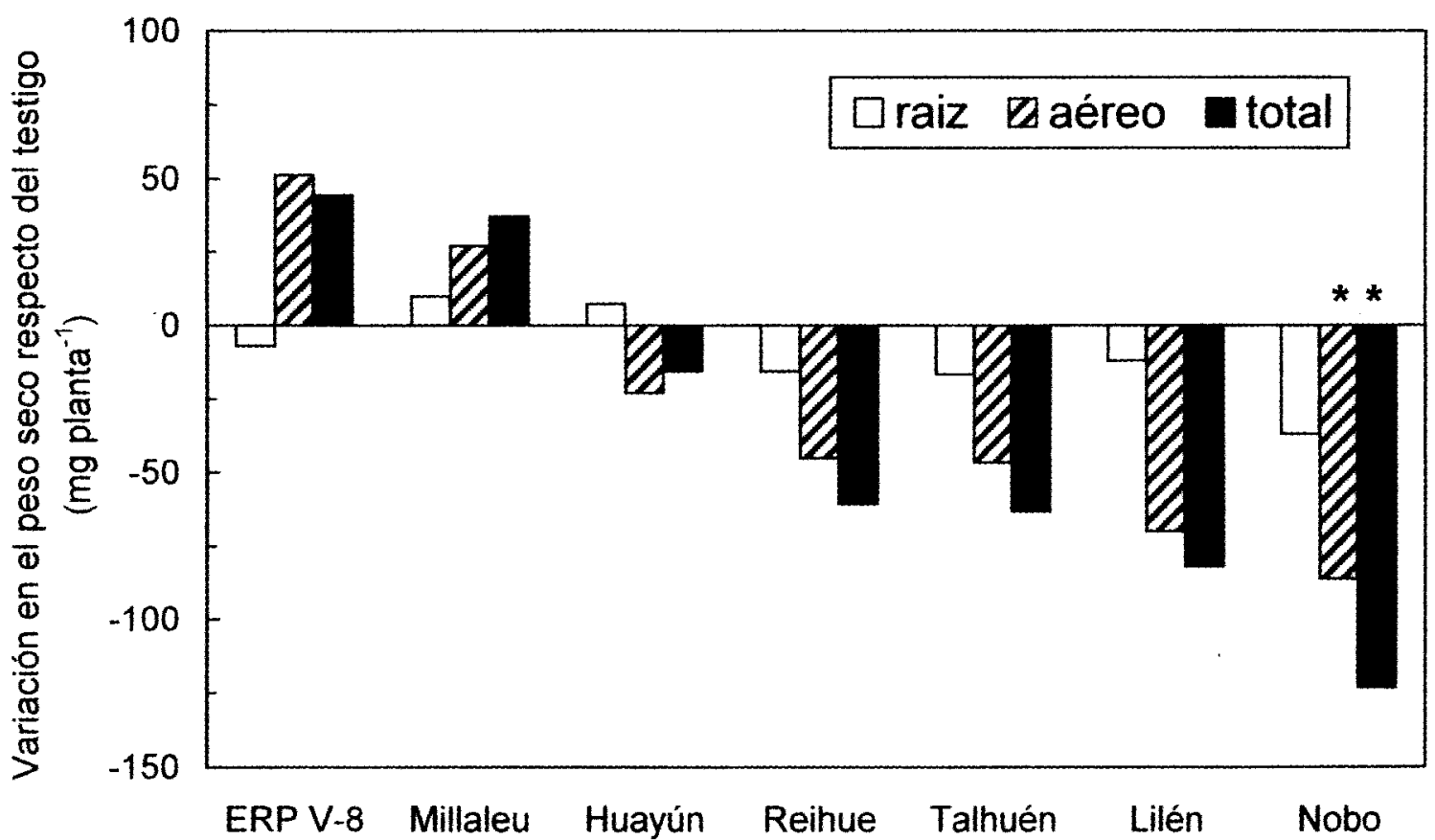

Fig. 3. Efecto de la radiación UV-B sobre el peso seco de siete variedades de trigo al estado de $5^{\mathbf{a}}$ hoja. Los asteriscos indican que la reducción en el peso seco de las plantas tratadas respecto de los testigos fue significativa $(\mathrm{P}<0,05 ; \mathrm{n}=28)$.

Effect of the UV-B irradiation on dry weight of seven wheat varieties, at the $5^{\text {th }}$ leaf state. Asterisks, indicate significant reductions in weight of the UV-B treated plants with respect to the non UV-B treated plants $(P<0.05 ; n=28)$. 
UV-B alcanzó un $16 \%$ con respecto de las no irradiadas.

\section{Efecto sobre la Asimilación de $\mathrm{CO}_{2}$}

La asimilación neta de $\mathrm{CO}_{2}$ se midió, luego de terminado el período de irradiación con UV-B, bajo una intensidad luminosa similar a la cual las plantas fueron cultivadas $\left(400 \mu \mathrm{mol} \mathrm{m} \mathrm{m}^{-2} \mathrm{~s}^{-1}\right)$. Los resultados indican que bajo estas condiciones, la irradiación con UV-B produce un efecto inhibitorio en la tasa de asimilación neta de $\mathrm{CO}_{2}$ en todas las variedades que en promedio fue $12,3 \%$. Esta inhibición fue particularmente importante en el caso de Lilén y Talhuén donde la disminución en relación a las plantas no irradiadas con UV-B fueron de $17,9 \%$ y $25,1 \%$ respectivamente. Sin embargo, el efecto alcanza solo significancia estadística en Talhuén (Tabla 1). Las variedades en que la asimilación de $\mathrm{CO}_{2}$ fue menos afectada fueron Huayún, ERP V-8 y Nobo. Curiosamente en la variedad Nobo, en la cual la irradiación con UV-B produjo una importante disminución de la materia seca, la tasa de asimilación neta de $\mathrm{CO}_{2}$ prácticamente no se afectó. Además cabe señalar que las plantas no irradiadas con UV-B de esta misma variedad presentaron a su vez las tasas más bajas de asimilación de $\mathrm{CO}_{2}$.

Efecto sobre los pigmentos absorbedores $U V-B$

Al término del experimento, las plantas no irradiadas con UV-B, presentaron un contenido simi- lar de compuestos absorbedores de UV a excepción de la variedad Nobo que presentó un alto contenido de estos compuestos (Fig. 4). En cambio, las plantas de todas las variedades irradiadas con UV-B, mostraron un significativo aumento de estos pigmentos expresado como absorbancia $(300 \mathrm{~nm})$ por unidad de área foliar $\left(A_{300} \mathrm{~cm}^{-2}\right)$. Sin embargo, la magnitud de este aumento no fue igual en todos los casos, observándose en orden decreciente los siguientes incrementos relativos: Lilén (30,8\%), Millaleu $(23,3 \%)$, Talhuén $(23,3 \%)$, Nobo $(21,6 \%)$, Huayún $(20,3 \%)$, ERP V-8 (17,6\%) y Reihue $(17,5 \%)$. Dado que Nobo fue la variedad con mayor contenido original de estos compuestos en plantas no irradiadas y una de las variedades con mayor incremento relativo luego del tratamiento con UV-B $(21,6 \%)$, fue también aquella que presentó el mayor contenido real de estos compuestos al final del experimento (Fig. 4).

\section{Efecto sobre la eficiencia fotoquímica del PSII}

En todos los casos los valores de la eficiencia fotoquímica del PSII (Fv/Fm) obtenidos en hojas de plantas no irradiadas con UV-B y cultivadas en baja intensidad luminosa $\left(400 \mu \mathrm{mol} \mathrm{m} \mathrm{m}^{-2} \mathrm{~s}^{-1}\right)$, estuvieron, dentro del rango considerado normal para una planta no estresada $(>0,79)$, (Demmig \& Bjorkman, 1987). Las diversas variedades no presentaron diferencias significativas entre ellas (Fig. 5a) y contrariamente a lo esperado, en todos los casos hubo un aumento en la eficiencia, que pese

\section{TABLA 1}

Efecto de la radiación UV-B sobre la asimilación neta de $\mathrm{CO}_{2}\left(\mu \mathrm{mol} \mathrm{m}^{-2} \mathrm{~s}^{-1}\right)$ en siete variedades de trigo. Letras minúsculas distintas, indican diferencias significativas entre tratamientos. Letras mayúsculas distintas indican diferencias significativas entre variedades ( $P$

$$
<0,05 ; \mathrm{n}=8 \text { ) }
$$

Effect of the UV-B radiation on the maximum $\mathrm{CO}_{2}$ assimilation ( $\mu$ mol $\mathrm{m}^{-2} \mathrm{~s}^{-1}$ ) of seven wheat varieties. Different lower case letters, indicate significant differences between treatments. Different capital letters indicate significant differences between varieties $(\mathrm{P}<0.05 ; \mathrm{n}=8)$

\begin{tabular}{lccr}
\hline Variedades & \multicolumn{3}{c}{$\begin{array}{c}\text { Asimilación neta máxima de } \mathrm{CO}_{2}\left(\mu \mathrm{mol} \mathrm{m}^{-2} \mathrm{~s}^{-1}\right) \\
\text { Variación respecto del testigo }(\%)\end{array}$} \\
\hline ERP-V8 & UV-B (Testigo) & $14,69 \mathrm{aA}$ & $-5,4$ \\
Lilén & $15,53 \mathrm{aA}$ & $11,86 \mathrm{aAB}$ & $-17,9$ \\
Millaleu & $14,45 \mathrm{aA}$ & $11,99 \mathrm{aAB}$ & $-12,2$ \\
Nobo & $13,66 \mathrm{aA}$ & $9,38 \mathrm{a} \mathrm{B}$ & $-4,9$ \\
Reihue & $9,86 \mathrm{a} \mathrm{B}$ & $12,39 \mathrm{aAB}$ & $-10,4$ \\
Talhuén & $13,83 \mathrm{aA}$ & $12,03 \mathrm{bAB}$ & $-25,1$ \\
Huayún & $16,07 \mathrm{aA}$ & $12,33 \mathrm{aAB}$ & $-6,1$ \\
& $13,13 \mathrm{aAB}$ & & $-12,3$ \\
Promedio & $13,79 \mathrm{a}$ & $12,10 \mathrm{a}$ &
\end{tabular}




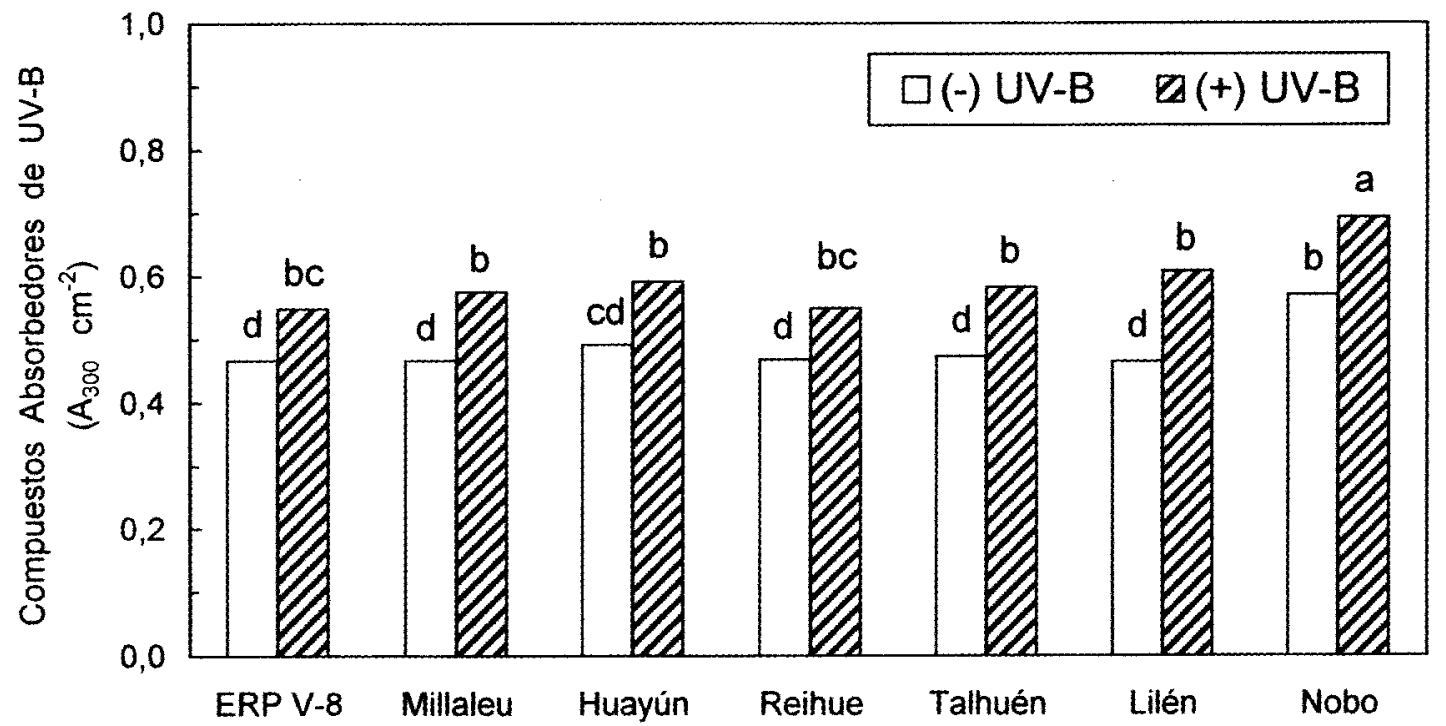

Fig. 4. Contenido de compuestos absorbedores de radiación UV-B $\left(\mathrm{A}_{300} \mathrm{~cm}^{-2}\right)$, en plantas irradiadas y no irradiadas de siete variedades de trigo. Letras distintas indican diferencias significativas entre plantas tratadas y no tratadas con radiación UV-B $(\mathrm{P}<0,05 ; \mathrm{n}=8)$.

Content of UV-B absorbing compounds $\left(A_{300} \mathrm{~cm}^{-2}\right)$ in UV-B treated and untreated plants of seven wheat varieties. Different letters indicate significant differences between varieties and treatments $(P<0.05 ; n=8)$.

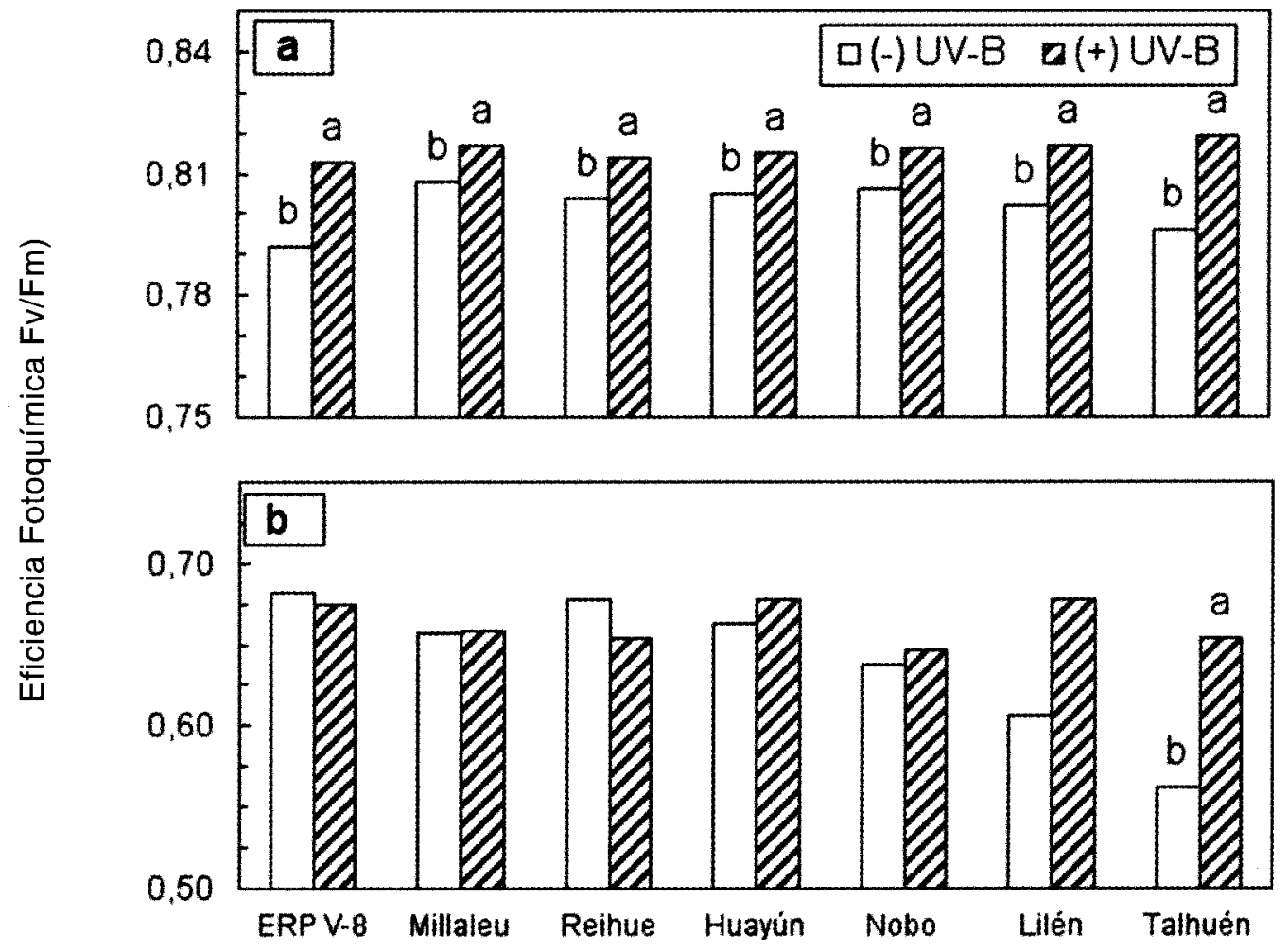

Fig. 5. Efecto de la radiación UV-B sobre la eficiencia fotoquímica ( $\mathrm{Fv} / \mathrm{Fm})$, de siete variedades de trigo, medido en plantas que crecieron en (a) baja intensidad luminosa $\left(400 \mu \mathrm{mol} \mathrm{m}^{-2} \mathrm{~s}^{-1}\right)$ y (b) luego de

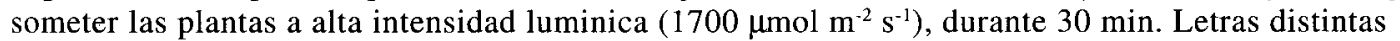
indican diferencias significativas entre tratamientos para una misma variedad $(\mathrm{P}<0,05 ; \mathrm{n}=12)$.

Effect of the UV-B irradiation on the photochemical efficiency ( Fv/Fm) of the seven wheat varieties, measured in plants

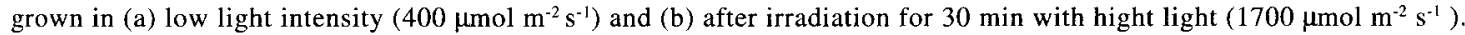
Different letters, indicate significant differences between treatments for the same variety $(\mathrm{P}<0.05 ; \mathrm{n}=12)$. 
a ser pequeño, resultó ser significativo respecto de las plantas no irradiadas, debido a la muy baja variabilidad de los datos. Las plantas que fueron cultivadas a baja intensidad luminosa presentaron valores de Fv/Fm inferiores a 0,7 cuando se sometieron, por un período de 30 minutos, a una

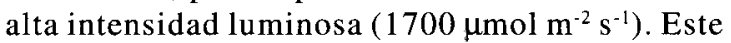
valor, que resultó ser significativamente menor a los obtenidos sin este tratamiento, revela que la alta intensidad luminosa indujo en todas las variedades un alto grado de fotoinhibición (Fig. $5 b)$. Sin embargo, en las plantas no irradiadas con UV-B de las variedades Lilén y Talhuén este efecto fue claramente más pronunciado. En ambos casos los valores de Fv/Fm fueron bastante menores que los del resto de las variedades: $0,6 \mathrm{y}$ 0,56 respectivamente, lo que indica que estas variedades son también más sensibles a la fotoinhibición por radiación visible que el resto. Sin embargo, en plantas de estas mismas variedades, previamente irradiadas con radiación UV-B y luego de ser expuestas a alta intensidad de radiación visible, la eficiencia fotoquímica del PSII, fue mayor a la de las plantas no irradiadas con UV-B. Esto es particularmente notorio en la variedad Talhuén donde estas diferencias alcanzan niveles significativos (Fig. 5b).

\section{DISCUSION}

Bajo las condiciones del presente estudio, se puede indicar que entre todas las variedades estudiadas: Talhuén, Lilén y Nobo fueron las más sensibles al tratamiento con radiación UV-B. Esto se puede concluir al considerar principalmente los efectos de esta radiación sobre los parámetros de crecimiento de estas tres variedades. Así por ejemplo, la reducción del área foliar en estas variedades fue importante, indicando que tal como en otras especies (Dickson \& Caldwell 1978, Dellenburg et al. 1995, González et al. 1998, Pinto et al. 1999), en trigo este proceso es también sensible a la radiación UV-B. La expansión foliar se ve afectada por el efecto de la radiación UV-B tanto sobre la división como sobre la expansión celular (Biggs et al. 1981, Tevini et al. 1981, Cen \& Bornman 1990). En el caso de este estudio no es posible determinar exactamente cual fue la causa de la reducción en el área foliar en las variedades más sensibles. Aunque por el momento en que se detectó ( $4^{a}-5^{a}$ semana después de emergencia), se podría pensar que la expansión celular fue la más afectada. Esto debido a que en plántulas de cereales la división celular estaría esencialmente centrada en los meristemas basales y ocurriría durante la primera semana después de emergencia, cuando las hojas están aún dentro del coleoptilo y por lo tanto, probablemente con sus meristemos protegidos de la radiación UV-B (Liu et al. 1995). Con posterioridad a la división celular ( $2^{\mathrm{a}}$ semana en adelante), sería la expansión celular el proceso que más controlaría el crecimiento de las hojas y por lo tanto, el que más podría haberse afectado en el caso de este estudio.

En general los resultados obtenidos hacen pensar que en las variedades más sensibles, la disminución del área foliar producida por la irradiación con UV-B pudo haber influido más que la disminución de la tasa de asimilación neta de $\mathrm{CO}_{2}$ en la acumulación de la biomasa (Fig. 3). Esto posiblemente a través de la reducción en la intercepción total de la radiación fotosintéticamente activa y por lo tanto, del total de $\mathrm{CO}_{2}$ asimilado por la planta durante el período de crecimiento. Este efecto indirecto sobre la acumulación de materia seca, parece particularmente aplicable a la variedad Nobo en la cual la irradiación con UV-B prácticamente no afectó la tasa de asimilación neta de $\mathrm{CO}_{2}$ por unidad de área (Tabla 1), pero sí el área foliar total. Esta variedad, junto con presentar la más alta reducción en la acumulación de materia seca total (130 mg planta ${ }^{-1}$, Fig. 3) también presentó la más alta reducción en área foliar: $24,5 \mathrm{~cm}^{2}$ planta ${ }^{-1}$, (Fig. 1). En las otras dos variedades con altas reducciones en la acumulación de materia seca (Lilén y Talhuén), la disminución en área foliar también fue importante: 18,3 y 18,55 $\mathrm{cm}^{2}$ planta $^{-1}$ respectivamente, (Fig. 1) aunque en la variedad Talhuén, el $25 \%$ de reducción observado en la tasa de asimilación neta de $\mathrm{CO}_{2}$ por unidad de área foliar (Tabla 1), pudo también haber influido en esta reducción. Así, una reducción en la acumulación de materia seca no necesariamente resulta de una reducción en la asimilación neta del carbono por unidad de área, siendo varios los estudios que señalan que es, a través de la reducción del área foliar, que la radiación UV$B$ podría ejercer su más importante acción sobre la acumulación de la materia seca (Barnes et al. 1990, Sullivan et al. 1996, González et al. 1998, Pinto et al. 1999). Esto puede suceder especialmente cuando los niveles de UV-B aplicados no son elevados o son suficientemente atenuados por los compuestos de la epidermis foliar impidiendo que los mecanismos fotosintéticos se afecten. Así, por ejemplo, en el caso de la variedad Nobo, los niveles de compuestos absorbedores de UV$B$, tanto en las plantas irradiadas como en las no irradiadas con UV-B, fueron los más elevados entre todas las variedades estudiadas (Fig. 4). Por lo que se podría inferir que en este caso existió una mayor atenuación de la radiación UV-B en la 
epidermis, produciéndose una mayor protección del aparato fotosintético que en las otras variedades. En el otro extremo se encontraría la variedad Talhuén con una importante reducción en la asimilación neta de $\mathrm{CO}_{2}$ por causa de la radiación UV-B $(25 \%)$ y a su vez con uno de los menores niveles de compuestos absorbedores de UV-B en las plantas irradiadas (Fig. 3). En este caso, la radiación UV-B, además de afectar la expansión del área foliar y porque posiblemente penetró más fácilmente hasta los tejidos del mesófilo, alteró también algunos de los mecanismos relacionados con la asimilación del $\mathrm{CO}_{2}$. Lilén, la otra variedad considerada sensible a UV-B, debido a su reducción en el área foliar y la materia seca, se podría decir que se encuentra en una situación intermedia respecto de su aumento en compuestos absorbedores de UV y de su disminución de la asimilación de $\mathrm{CO}_{2}$.

En relación con la disminución del peso fresco total, las tendencias son similares a las encontradas para el caso de la materia seca. Los mismos genotipos (Nobo, Talhuén y Lilén) son los que presentan la mayor disminución del peso fresco total, no observándose variaciones significativas en el resto de los genotipos. De acuerdo con este parámetro, nuevamente es la variedad Nobo la que presenta la mayor sensibilidad, disminuyendo $13,4 \%$ de su peso total como materia seca y $86,6 \%$ como agua. En cambio Talhuén y Lilén presentaron mayores disminuciones relativas de agua: 94 y $89 \%$ respectivamente. Esta disminución en el contenido de agua en los tejidos de plantas irradiadas con UV, fue de similar magnitud (93\% en promedio) en las variedades clasificadas como más resistentes a esta radiación, por lo que se podría inferir que la diferencia con las variedades más sensibles fue esencialmente dada por la capacidad de las más resistentes en mantener relativamente estable la síntesis de materia seca. En efecto, los dos mecanismos señalados aquí como responsables de la acumulación de la materia seca: el área foliar y la asimilación de $\mathrm{CO}_{2}$ por unidad de área, en el grupo de las variedades resistentes tuvieron alteraciones por la radiación UV-B, bastantes menores que las observadas en el grupo de las más sensibles. Relacionar esta menor alteración en el crecimiento con una mayor concentración de compuestos absorbedores de UV, en el caso de las variedades más resistentes no parece posible ya que en este caso el incremento observado fue similar a aquel observado en las variedades más sensibles.

Un aumento en la concentración de los compuestos absorbedores de radiación UV-B, por acción de esta radiación, no necesariamente implica una mayor síntesis de éstos. Aunque varios son los trabajos que indican que la radiación UV$\mathrm{B}$ induce la síntesis de novo de estos compuestos, en especial de flavonoides (Beggs \& Wellmann 1994) aparentemente esta no siempre se produce en tejidos verdes y maduros (Wellmann, comunicación personal). Un aumento en la concentración de estos compuestos puede ser producto de una disminución en el tamaño de las células y en consecuencia del área foliar. Por lo tanto, el au-

TABLA 2

Efecto de la radiación UV-B sobre el contenido de Compuestos Absorbedores UV-B por planta $\left(A_{300}\right.$ planta) en siete variedades de trigo. Letras minúsculas distintas indican diferencias significativas entre tratamientos. Letras mayúsculas distintas indican diferencias significativas entre variedades $(P<0,05 ; n=8)$

Effect of UV-B radiation on the UV-Absorbing Compounds ( $A_{300}$ plant) in of seven wheat varieties. Different lower case letters, indicate significant differences between treatments. Different capital letters indicate significant differences between varieties $(\mathrm{P}<0.05 ; \mathrm{n}=8)$

\begin{tabular}{|c|c|c|c|}
\hline \multirow[t]{2}{*}{ Variedades } & \multicolumn{3}{|c|}{ Absorbancia $(300 \mathrm{~nm}) /$ planta } \\
\hline & - UV-B (Testigo) & + UV-B & Variación respecto del testigo (\%) \\
\hline Nobo & 70,8 a $\mathrm{A}$ & 68,8 a A & $-2,8$ \\
\hline Talhuén & 51,6 a $\mathrm{BC}$ & 52,6 a $\mathrm{BC}$ & $+1,9$ \\
\hline Reihue & 39,5 a $E$ & 42,2 a $E$ & $+6,4$ \\
\hline Lilén & 48,4 a CD & 52,1 a $\mathrm{CD}$ & $+7,6$ \\
\hline Millaleu & 40,2 a $E$ & $44,7 \mathrm{a} \quad \mathrm{DE}$ & $+10,1$ \\
\hline Huayún & 55,2 b $\quad$ B & 62,2 a $\mathrm{A}$ & $+11,3$ \\
\hline ERP-V8 & $43,9 \mathrm{~b}$ DE & 52,0 a $\quad \mathrm{BC}$ & $+15,6$ \\
\hline Promedio & $49,9 \mathrm{a}$ & $53,5 \mathrm{a}$ & $+7,2$ \\
\hline
\end{tabular}


mento observado en la concentración $\left(A_{300} \mathrm{~cm}^{-2}\right)$ en las plantas irradiadas con UV-B de las variedades más sensibles (Nobo, Lilén y Talhuén), puede que efectivamente sea el producto de la menor expansión foliar observada. Sin embargo, en el caso de las variedades más resistentes (ERP V-8, Huayún, Reihue y Millaleu), en las que no se detectaron cambios significativos en -el área foliar, puede que este aumento se deba más a una inducción de síntesis. En efecto, al expresar los niveles de compuestos absorbedores en absorbancia por planta $\left(A_{300}\right.$ planta $\left.^{-1}\right)$ (Tabla 2$)$ se observa que los contenidos de estos compuestos aumentan significativamente en las plantas irradiadas de las variedades resistentes (Millaleu, Huayún y ERP-V8) en cambio en las variedades más sensibles (Nobo y Talhuén), el contenido por planta prácticamente no cambia con el tratamiento con radiación UV-B. Los resultados de este ensayo no permiten avanzar más en el análisis del rol protector de los compuestos ábsorbedores de UV en el trigo por lo que más estudios son necesarios en esta especie.

En relación con el efecto de la radiación UV-B sobre la eficiencia fotoquímica de la fotosíntesis (Fv/Fm) se observa que en condiciones de baja intensidad luminosa los valores son bastante elevados tanto en plantas no irradiadas como en las irradiadas con UV-B, mostrando que el tratamiento con UV-B no afectó negativamente el transporte de electrones en el PSII. Por el contrario, el incremento observado podría indicar que la dosis de radiación UV-B aplicada más bien mejora esta eficiencia en todos los genotipos. En función de estos resultados, se podría especular sobre el rol protector de los compuestos absorbedores de UV-B, cuya concentración aumentó en todos los casos de plantas irradiadas. Sin embargo, estos resultados se contraponen a las disminuciones encontradas en la asimilación de $\mathrm{CO}_{2}$ en las plantas irradiadas con UV-B, indicando que es probable que otros mecanismos alternativos para el consumo de electrones se hayan activado con el tratamiento UV-B y que hayan contribuido a mantener alta esta eficiencia aún con una baja tasa en la asimilación de $\mathrm{CO}_{2}$ (Allen et al. 1997). De todas maneras, más estudios a este respecto son necesarios para poder sacar conclusiones más definitivas.

Al pretratar las plantas por media hora con alta intensidad de radiación visible $\left(1700 \mu \mathrm{mol} \mathrm{m} \mathrm{m}^{-2} \mathrm{~s}^{-1}\right)$, los valores Fv/Fm encontrados señalan un alto grado de fotoinhibición en todas las variedades. Un caso interesante es lo que sucede en las dos variedades clasificadas como más sensibles: Talhuén y Lilén, en que la radiación UV-B hace que la fotoinhibición sea menos drástica que en las plantas no irradiadas con UV-B (mayores Fv/ Fm en plantas irradiadas con UV-B que en plantas no irradiadas). Esta especie de fotoprotección inducida por la radiación UV-B, es interesante ya que se produce en las variedades que a baja intensidad luminosa se revelaron sensibles a esta radiación (disminución del área foliar y de la tasa de asimilación de $\mathrm{CO}_{2}$ ). Se ha postulado, que tanto el daño producido en el aparato fotosintético por fotohibición por radiación visible como por radiación UV, es en gran medida producto de la generación de especies activas de $\mathrm{O}_{2}$ (Hideg \& Vass 1996, Barabás et al. 1998) y que la activación de mecanismos detoxificadores en los cloroplastos ayudaría a los vegetales a soportar estos estreses oxidativos (Noctor \& Foyer 1998). Por lo tanto, es posible que la inducción por UV$B$ de fotoprotección a la radiación visible en trigo, sea en este caso consecuencia de una activación de los mecanismos de detoxificación a causa de la aplicación previa de la radiación UV$\mathrm{B}$, los cuales estarían ya funcionando al momento de la aplicación de alta intensidad luminosa. Inducción cruzada de tolerancia a altas intensidades de radiación visible mediante aplicaciones previas de UV-B han sido ya reportadas en frejol (Pinto et al. 1999) y esta sería la primera vez que se reporta en trigo.

Finalmente se concluye que las variedades ERP V-8, Millaleu y Huayún son las que soportaron mejor el tratamiento con UV-B impuesto, sugiriendo que al menos durante los primeros estados de desarrollo, su crecimiento no se alteraría mayormente a causa de dosis de UV-B similares a las usadas en este estudio. Estas dosis corresponderían aproximadamente entre un 15 a un $20 \%$ superior a las observadas en forma natural durante el período de crecimiento del trigo en la zona Central de Chile. Sin embargo, de acuerdo a mediciones efectuadas en el Norte (datos no publicados) estas son muy similares a las encontradas, para igual período, en la III Región. Por lo tanto y solo considerando este factor, es posible que las variedades más tolerantes (ERP V-8, Millaleu y Huayún) se adapten más a las condiciones de radiación UV-B del Norte del país que las más sensibles (Lilén, Talhuén y Nobo). Esta últimas podrían, por lo tanto, prosperar mejor bajo las condiciones de radiación UV-B de la zona Centro - Sur, aunque para tener una opinión más definitiva a este respecto, futuros estudios deberían también considerar la interacción con otros factores ambientales, otros estados de desarrollo y la repercución del incremento de la radiación UV-B en la producción final de grano. 


\section{AGRADECIMIENTOS}

Este trabajo fue financiado con aportes del Departamento de Investigación y Desarrollo de la Universidad de Chile y del Proyecto Fondecyt 1950860.

\section{LITERATURA CITADA}

ALLEN DJ, IF MCKEE, PK FARAGE \& NR BAKER (1997) Analysis of limitations to $\mathrm{CO}_{2}$ assimilation on exposure of leaves of two Brassica napus cultivars to UV-B. Plant Cell and Environment 20: 633-640.

BARABAS KN, T SZEGLETES ZSOL,A PESTENACZ. K FÜLÖP \& L ERDEI (1998) Effects of excess UV$B$ irradiation on the antioxidant defence mechanisms in wheat (Triticum aestivum L.) seedlings. Journal of Plant Physiology 153: 146-153.

BARNES PW, SD FLINT \& MM CALDWELL (1990) Morphological responses of crop and weed species of different growth forms to ultraviolet-B radiation. American Journal of Botany 77: 1354-1360.

BECWAR MR, FD III MOORE \& MJ BURKE (1982) Effects of deletion and enhancement of ultraviolet-B $(280-315 \mathrm{~nm})$ radiation on plants grown at $3000 \mathrm{~m}$ elevation. Hort Science 107: 771:779.

BEGGS CJ \& E WELLMANN (1994) Photocontrol of flavonoid biosynthesis. En: Kendrick RE \& GHM Kronenberg (eds) Photomorphogenesis in plants: 733 751, Kluwer Academic Publishers, Dordrecht.

BIGGS RH, SV KOSSUTH \& AH TERAMURA (1981) Response of 19 cultivars of soybean to ultraviolet $B$ irradiance. Physiologia Plantarum 53: 19-26.

BORNMAN JF (1989) Target sites of UV-B radiation in photosynthesis of higher plants. Journal of Photochemistry and Photobiology 4: 145-158.

BORNMAN JF \& TC VOGUELMANN (1991) The effect of UV-B radiation on leaf optical properties measured with fiber optics. Journal of Experimental Botany 42: 547-554.

CABRERA S, S BOZZO \& H FUENZALIDA (1995) Variations in UV radiation in Chile. Photochemistry and Photobiology 28: 137-142.

CEN YP \& JF BORNMAN (1990) The response of bean plants to UV-B radiation under different irradiances of background visible light. Journal of Experimental Botany 41: 1489-1495.

DAY TA (1993) Relating UV-B radiation screening effectiveness of foliage to absorbing-compound concentration and anatomical characteristics in a diverse group of plants. Oecologia 95: 542-550.

DAY TA \& TC VOGUELMAN (1995) Alteration in phothosynthesis and pigment distribution in pea leaves following UV-B exposure. Physiologia Plantarum 94 : 433-440.

DAY TA, TC VOGELMANN \& EH DE LUCIA (1992) Are some plant life forms more effective than others in screening out ultraviolet-B radiation? Oecologia 92: 513-519.
DAY TA, BW HOWELLS \& CT RUHLAND (1996) Changes in growth and pigment concentrations with leaf age in pea under modulated UV-B radiation field treatments. Plant Cell and Environment 19: 101-108.

DELLEMBURG LR, JH SULLIVAN \& AH TERAMURA (1995) Leaf expansion and development of photosynthetic capacity and pigments in Liquidambar styraciflua (Hamamelidaceae) - effects of UV-B radiation. American Journal of Botany 82: 878-885.

DEMMIG B \& O BJÖRKMAN (1987) Comparison of excessive light on chlorophyll fluorescence $(77 \mathrm{~K})$ and photon yield of $\mathrm{O}_{2}$ evolution in leaves of higher plants. Planta 171: 171-184.

DICKSON JD, \& MM CALDWELL (1978) Leaf development of Rumex patientia L. (Polygonaceae) exposed to UV irradiation $(280-320 \mathrm{~nm})$. American Journal of Botany 55: 857-863.

FELDHEIM K \& JK CONNER (1996) The effects of increased UV-B radiation on growth, pollination sucess and lifetime female fitness in two Brassica species. Oecología 106: 284-297.

FISCUS EL \& FL BOOKER (1995) Is increased UV-B a threat to crop photosynthesis and productivity? Photosynthesis Research 43: 81-92.

FREDERICK JE, HE SNELL \& EK HEYWOOD (1989) Solar ultraviolet radiation at the Earth's surface. Photochemestry and Photobiology 50: $443-450$.

GENTY B, JM BRAINTAIS \& NR BAKER (1989) The relationship between the quantum yield of photosynthetic electron transport and quenching of chlorophyll fluorescence. Biochimica and Biophysica Acta 990: 87 - 92

GONZALEZ R, R MEPSTED, AR WELLBURN \& ND PAUL (1998) Non-photosynthetic mechanisms of growth reduction in pea (Pisum sativum $\mathrm{L}$.) exposed to UV-B radiation. Plant Cell and Environment 21:23 -32 .

HIDEG E, \& I VASS (1996) UV-B induced free radical production in plant leaves and isolated thylakoid membranes. Plant Science 115: $251-260$.

HOAGLAND DR \& DI ARNON (1950) The water culture method for growing plants without soil. Berkeley. California Agricultural experiment station. The College of Agriculture University of California. Circular $\mathrm{N}^{\circ} 347$.

HUANG LK, J HE, WS CHOW, MI WHITECROSS \& JM ANDERSON (1993) Responses of detached rice leaves (Oryza sativa L.) to moderate supplementary ultraviolet- $B$ radiation represent an initial method to determine relative sensitivity to ultraviolet-B irradiation. Australian Journal of Plant Physiology 20: 285-297.

JANSEN MAK, V GABA \& B GREENBERG (1998) Higher plants and UV-B radiation: balancing damage, repair and acclimation. Trends in Plant Science 3: 131-135.

KIRCHHOFF VWJH, F ZAMORANO \& C CASICCIA (1997) UV-B enhancements at Punta Arenas, Chile. Journal of Photochemistry and Photobiology B: Biology 38: 174-177.

KRAUSE G \& E WEIS (1984) Chlorophyll fluorescence as a tool in plant physiology II. Interpretation of fluorescence signals. Photosynthesis Research 5: 139157. 
KULANDAIVELU G, S MARAGATHAM \& N NEDUNCHEZHIAN (1989) On the possible control of Ultraviolet-B induced response in growth and photosynthetic activities in higher plants. Physiologia Plantarum 76: 398-404.

LIU L, GITZ DCIII \& JW MCCLURE (1995) Effects of UV-B on flavonoids, ferulic acid, growth and photosynthesis in barley primary leaves. Physiologia Plantarum 93: 725-733.

MILLER JE, FL BOOKER, EL FISCUS, AS HEAGLE, WA PURSLEY, S VOZZOS \& WW HECK (1994) Ultraviolet-B radiation and ozone effects on growth, yicld and photosynthesis of soybean. Journal of Environmental Quality 23: 83-91.

NEDUNCHEZHIAN N \& G KULANDAIVELU (1991) Evidence for the Ultravioleta-B $(280-320 \mathrm{~nm})$ radiation induced structural reorganization and damage of photosystem II polypeptides in isolated chloroplasts. Physiologia Plantarum 81: 558-562.

NOCTOR G \& KH FOYER (1998) Ascorbate and glutathione: keeping active oxygen under control. Annual Review of Plant Physiology \& Plant Molecular Biology 49: 249-279.

PARKINSON KJ, W DAY \& JE LEACH (1980) Aportable system for measuring the photosynthesis and transpiration of graminaceous leaves. Journal of Experimental Botany 31: 1441-1453.

PINTO ME, P CASATI, H TSUI-PING, MSB KU \& GE EDWARDS (1999) Effects of UV-B radiation on growth, photosynthesis, UV-B absorbing compounds and NADP-malic enzyme in bean (Phaseolus vulgaris L.) grown under different nitrogen conditions. Journal of Photochemistry and Photobiology B: Biology 48: $200-209$.

ROY G, P GIES \& G ELLIOT (1990) Ozone depletion. Nature 347: 50-56

SHARMA PK, P ANAND, S SANKHALKAR \& $R$ SHETYE (1998) Photochemical and biochemical changes in wheat seedlings exposed to supplementary ultraviolet-B radiation. Plant Science 132: 21-30.

STRID A \& RJ PORRA (1992) Alteration in pigment content in leaves of Pisum sativum after exposure to supplementary UV-B. Plant and Cell Physiology 33: 1015-1023

STRID A, CHOWW S \& ANDERSON JM (1990) Effects of suplementary ultraviolet-B radiation on photosynthesis in pisium sativum. Biophysica and Biochimica Acta 1020: 260-268

STRID A, WS CHOW \& JM ANDERSON (1994) UV-B damage and protection at the molecular level in plants. Photosynthesis Research 43: 81-92.
SULLIVAN JH \& AH TERAMURA (1990) Field study of the interaction between solar Ultraviolet-B radiation and drought on photosynthesis and growth in soybean. Plant Physiology 92: 141-146.

SULLIVAN JH, BW HOWELLS, CT RUHLAN \& TA DAY (1996) Changes in leaf expansion and epidermal screening effectiveness in Liquidambar styraciflua and Pinus taeda in response to UV-B radiation. Physiologia Plantarum 98: 349-357.

TERAMURA AH (1983) Effects of ultraviolet-B radiation on the growth and yield of crop plants. Physiologia Plantarum 58: 415-427.

TERAMURA AH (1990) Implications of stratospheric ozone depletion upon plant production. Hort Science 25: 1557-1559.

TERAMURA AH \& JH SULLIVAN (1987) Soybean growth responses to enhanced levels of ultraviolet-B radiation under greenhouse conditions. American Journal of Botany 74: 975-979.

TERAMURA AH \& JH SULLIVAN (1994) Effects of UV$B$ radiation on photosynthesis and growth of terrestrial plants. Photosynthesis Research 39: 463-473.

TERAMURA AH, LH ZISKA \& AE SZTEIN (1991) Changes in growth and photosynthetic capacity of rice with increased UV-B radiation. Physiologia Plantarum 83: 373-380.

TEVINI M, IWANZIKW \& U THOMO (1981) Some effects of enhanced UV-B irradiation on the growth and composition of plants. Planta 153: 338-394.

TEVINI M, J BRAWN \& G FIESER (1991) The protective function of the epidermal layer of rye seedlings against UV-B radiation. Photochemistry and Photobiology 53: 329-333.

WILSON MI \& BM GREENBERG (1993) Protection of the D1 Photosystem reaction center protein from degradation in ultraviolet radiation following adaptation of Brassica napus L. to growth in ultraviolet-B. Photochemistry and Photobiology 57: 556-563.

ZENTHEN J, TN MIKKELSEN, G PALUDAN-MULLER \& H RO-PAULSEN (1997) Effects of increased UV$B$ radiation and elevated levels of tropospheric ozone on physiological processes in European beech (Fagus selvatica). Physiologia Plantarum 100: 281-290.

ZISKA LH \& AH TERAMURA (1992) CO2 enhancement of growth and photosynthesis in rice (Oryza sativa). Plant Physiology 99: 473481.

ZISKA LH, AH TERAMURA, JH SULLIVAN \& A McCOY (1993) Influence of ultraviolet-B (UV-B) radiation on photosynthetic and growth characteristics in field-grown cassava (Manihot esculentum Crantz). Plant Cell and Environment 16: 73-79. 